\title{
How well does the chiral expansion converge in nuclear and neutron matter?
}

\author{
Francesca Sammarruca* \\ University of Idaho, Moscow, Idaho, USA \\ E-mail: fsammarreuidaho.edu
}

\section{Luigi Coraggio}

Istituto Nazionale di Fisica Nucleare, Napoli, Italy

E-mail: coraggioena.infn.it

\section{Jeremy W. Holt}

University of Washington, Seattle, Washington, USA

E-mail: jwholtphys@gmail.com

\section{Nunzio Itaco}

Istituto Nazionale di Fisica Nucleare, Napoli, Italy

Seconda Universita' di Napoli

E-mail: itaco@na.infn.it

\section{Ruprecht Machleidt}

University of Idaho, Moscow, Idaho, USA

E-mail: machleideuidaho.edu

\section{Laura Elisa Marcucci}

Universita di Pisa, Pisa, Italy

Istituto Nazionale di Fisica Nucleare, Sezione di Pisa

E-mail: laura.elisa.marcuccieunipi.it

The equations of state of nuclear and neutron matter (and, more generally, neutron-rich matter) play a fundamental role towards the understanding of a broad spectrum of systems, ranging from the skins of neutron-rich nuclei to the structure of compact stars.

After a brief review of different approaches to predict the properties of nuclear and neutron matter, we will focus on error quantification in effective field theory. The various sources of uncertainty and their impact in infinite matter will be explored.

We will then report on recent calculations of the nuclear and neutron matter equations of state at different orders of the chiral expansion as well as changing resolution scale. We will discuss the significance of such predictions as a foundation for future studies of convergence of the chiral perturbation series.

The 8th International Workshop on Chiral Dynamics, CD2015 ***

29 June 2015 - 03 July 2015

Pisa,Italy

\footnotetext{
*Speaker.
} 


\section{Introduction and outline}

Microscopic nuclear physics seeks to describe the structure of nuclear systems in terms of the underlying few-nucleon forces, namely, $a b$ initio. Ideally, one wishes to derive nuclear forces from quantum chromodynamics (QCD), the fundamental theory of strong interactions. However, the well-known problem with QCD is that it is nonperturbative in the low-energy regime characteristic for nuclear physics, a fact which was perceived for a long time as an obstacle to a derivation of nuclear forces from QCD. The effective field theory (EFT) concept, chiral EFT in particular, has shown a way out of this problem and is presently a popular approach which starts from a lowenergy realization of QCD [1, 2]. In chiral EFT, one retains the basic degrees of freedom typical of low-energy nuclear physics, pions and nucleons, while fitting unresolved nuclear dynamics at short distances to the properties of two- and few-nucleon systems alone.

Although finite nuclei are a natural testing ground for many-body theories, infinite matter is an alternative and convenient system for testing such theories. Being translationally invariant and consisting of nucleons subjected only to their mutual strong interactions, it is theoretically simpler than a finite nucleus and yet, through its saturation properties, provides strong constraints for theoretical models of nuclear forces in the medium.

The chiral EFT approach, central to this paper, has been shown to produce realistic equations of state (EoS) for nuclear and neutron matter with the inclusion of repulsive three-nucleon forces (3NF) arising at order $\mathrm{N}^{2} \mathrm{LO}$ (next-to-next-to-leading order) in the chiral power counting $[3,4,5,6]$. In chiral EFT, the dominant two-pion-exchange component of this $3 \mathrm{NF}$ is constructed consistently with the $\mathrm{N}^{2} \mathrm{LO}$ two-body force.

The main purpose of this paper is to discuss error quantification in predictions of nucleonic matter obtained with chiral two- and three-body forces. The discussion will articulate through the following main points:

- We will start with a broad introduction and further underline the importance of studying infinite matter (symmetric or neutron-rich) as a testing ground for many-body theories.

- We will highlight two popular philosophies currently used to obtain predictions of nuclear and neutron matter properties, and comment on their respective advantages and disadvantages.

- We will then move to our focal point, namely, error quantification in chiral EFT, and address the main sources of uncertainty.

- We will present and discuss recent calculations of the energy per particle at different orders of chiral EFT. Prior to that, we will take the opportunity to review the development of the chiral two- and three-nucleon forces which we use.

- We will summarize our conclusions, work in progress, and future plans.

\section{Infinite matter: an important theoretical "laboratory"}

The typical outcome of nuclear matter investigations consists of the energy per particle and the pressure as functions of density (and additional variables, such as temperature, if appropriate). 
Adopting what is known as the "local density approximation", one can relate the EoS of infinite matter to finite systems. Vice versa, empirical information on nuclei have been extrapolated to an infinite system to provide constraints for theoretical models of nuclear matter.

When proton and neutron densities are different (that is, in the presence of isospin asymmetry), the energy per particle becomes a function of both the total density and the relative concentrations of neutrons and protons. The EoS of isospin-asymmetric matter naturally introduces the symmetry energy, similarly to the appearance of the symmetry term in the well-known Bethe-Weizsäcker formula. As will be discussed later in more details, the symmetry energy is typically approximated as the difference between the energy per particle of symmetric nuclear matter and pure neutron matter as a function of density.

Of particular contemporary interest is the EoS of highly neutron-rich matter, all the way to nearly pure neutron matter. This quantity is important for understanding wide ranging questions in modern nuclear structure physics, from the properties of rare isotopes to those of neutron stars. On the one hand, the density dependence of the symmetry energy is known to correlate strongly with the neutron skin thickness of a heavy nucleus. On the other hand, the pressure in neutron-rich matter is the main input for the structure equations of compact stars.

Therefore, microscopic predictions together with empirical constraints from observables that are sensitive to the EoS are an ideal combination to learn about the in-medium behavior of the nuclear force, particularly in isospin-asymmetric medium. We recall that the EoS is an important part of the input of transport models describing heavy-ion collisions and thus can be constrained through analyses of carefully selected observables in ion-ion scattering.

Concerning non-terrestrial observations, partnership between nuclear physics and astrophysics is increasingly important as better constraints on the high-density part of the EoS become available through more accurate measurements of neutron star masses.

In summary, studies of nucleonic matter are especially timely and important, as they support rich on-going and future experimental effort, both astrophysically and in terrestrial laboratories.

\section{Meson theory $v s$. chiral approaches: general considerations}

As mentioned earlier, the goal of microscopic nuclear physics is to derive the properties of nuclear systems from the basic few-nucleon interactions. Therefore, the first question to raise is how to derive those fundamental forces.

Meson-theoretic interactions, such as those obtained with one-boson-exchange models, have been, and still are, used extensively in nuclear structure calculations. At this point, it may be interesting to make some additional observations with regard to the structure of the nucleon-nucleon potential in chiral EFT as compared to the traditional meson-exchange model. In the latter, the pion, the intermediate-mass meson (typically, the fictitious $\sigma$ ), and the heavier bosons ( $\rho$ and $\omega$ ), provide the long-, the intermediate-, and the short-range behaviors, respectively, required by the phenomenology of the nuclear force. In chiral EFT, multi-pion exchange diagrams describe the longer and intermediate range parts of the force, whereas "contact terms" encode the short-range components. Both philosophies have in common the outstanding role of the pion. 


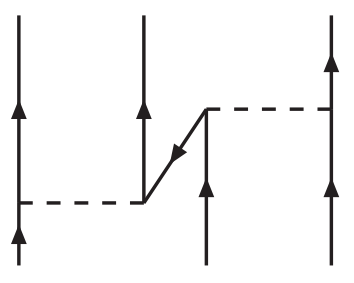

Figure 1: Three-nucleon force due to virtual excitation of a nucleon-antinucleon pair.

However, meson-exchange models (see, for instance, Ref. [7]) typically employ three-nucleon forces with little connection to the associated nucleon-nucleon (NN) force. The main problem is how to select the contributions to be retained in a systematic and model-independent way.

Relativistic approaches to nuclear matter have focused on the Dirac-Brueckner-Hartree-Fock (DBHF) method together with relativistic one-boson-exchange potentials [8], and are suitable to address a broad range of momenta and densities. This is desirable, since, in astrophysical contexts, the EoS of neutron-rich matter is required over many orders of magnitude in the nuclear density, potentially up to ten times that of saturated nuclear matter. The main strength of the DBHF framework is its ability to account in an efficient way for an important class of 3NF, namely virtual nucleon-antinucleon excitations, shown in Fig. 1, that lead to nuclear matter saturation properties close to the empirical density and energy [8].

In spite of the strong features offered by some meson-theoretic models, the problem shared with all non-EFT based approaches is that it is essentially impossible to estimate reliably the uncertainty associated with a particular prediction. On the other hand, EFT provides a well-defined framework to calculate observables where the truncation error decreases systematically as higher orders are included. More specifically, EFT is a theory in which the properties governed by lowenergy physics are specified by the choice of degrees of freedom and symmetries, and can be computed systematically. Short-range physics is included through the processes of regularization and renormalization. Together with an organizational scheme to rank-order the various contributions, known as power counting, two- and few-nucleon forces emerge on equal footing in a controlled hierarchy, as shown in Fig. 2.

\section{EFT uncertainty quantification}

Estimates of theoretical uncertainties [9] for calculations of the EoS have mostly focused on varying the low-energy constants and resolution scale at which nuclear dynamics are probed $[3,4$, $5,10,11,12]$. In recent work [13] we laid the foundation for order-by-order calculations of nuclear 


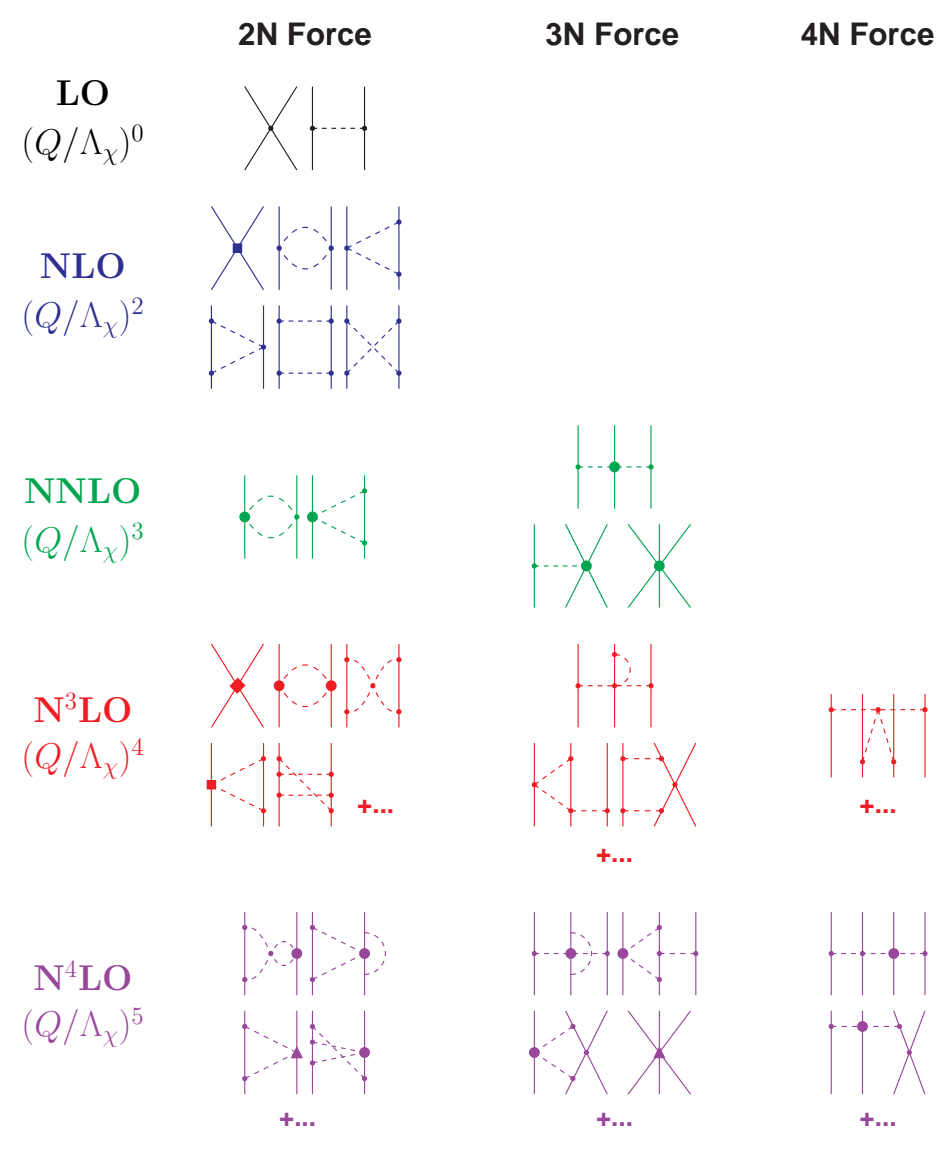

Figure 2: Chiral two- and few-nucleon forces up to 5th order of EFT.

many-body systems by presenting consistent NLO and $\mathrm{N}^{2} \mathrm{LO}$ chiral nuclear forces whose relevant short-range three-body forces are fit to $A=3$ binding energies and the lifetime of the triton. We then assessed the accuracy with which infinite nuclear matter properties and the isospin asymmetry energy can be predicted from order-by-order calculations in chiral EFT. Identifying the dominant sources of uncertainty in nuclear many-body calculations is an important open problem, especially as more stringent constraints on the EoS of neutron-rich matter and its density dependence are becoming available [14].

Uncertainty originates from:

- The choice of the many-body method (a source of error not inherent to EFT).

- Error in the determination of the low-energy constants (LECs). Short-range LECs (NN) and long-range LECs $(\pi \mathrm{N})$ must be considered separately.

- Regulator dependence.

- Truncation error.

In the following sections, we will address them systematically. 


\subsection{Uncertainty from the many-body method}

A variety of many-body methods are available and have been used extensively in nuclear matter. They include: the coupled-cluster method, many-body perturbation, variational Monte Carlo or Green's function Monte Carlo methods.

In computing the EoS, we employ the nonperturbative particle-particle ( $\mathrm{pp}$ ) ladder approximation. In the traditional hole-line expansion, it represents the leading-order contribution. We use the so-called continuous choice for the single particle potential, which is determined selfconsistently applying the effective mass approximation. To quantify the uncertainty carried by these choices, it is insightful to compare with Refs. [15, 16]. In Ref. [15], the authors report on coupled-cluster calculations in symmetric nuclear matter including pp and hole-hole (hh) diagrams (as well as an exact treatment of the Pauli operator). The overall effect, as seen from comparing the first and last entries in Table II of Ref. [15], is very small around saturation density, consistent with Table II in Ref. [5], and grows to $1.5 \mathrm{MeV}$ at the highest Fermi momentum included in the study. These calculations adopt the $\mathrm{N}^{3} \mathrm{LO}$ potential (with $\Lambda=500 \mathrm{MeV}$ ) and only two-nucleon forces. On the other hand, in Ref. [16] coupled-cluster calculations in nucleonic matter were performed at $\mathrm{N}^{2} \mathrm{LO}$ with two- and three-body forces and with the inclusion of selected triples clusters, namely correlations beyond pp and hh ladders. Note that triples signify what is known, in the more traditional language, as three-hole line contributions. The effects of these triples is found to be negligible in neutron matter and about $1 \mathrm{MeV}$ per nucleon in symmetric matter in the density range under consideration [16].

In the light of the above considerations, we conclude that a realistic estimate of the impact of using a nonperturbative approach beyond pp correlations is about $\pm 1 \mathrm{MeV}$ in nuclear matter around saturation density and much smaller in neutron matter. As we show below, such uncertainties are significantly smaller than those associated with variations in the cutoff scale.

\subsection{Uncertainty in the low-energy constants}

In order to quantify the error associated with possible variations of the (short-range) NN LECs, we refer to recent findings from the Granada group [17]. They applied 205 samples of smooth local potentials, all with $\chi^{2} /$ datum of approximately 1 , and found a variation of $\pm 15 \mathrm{keV}$ in the triton binding energy.

From our part, we performed Brueckner-Hartree-Fock calculations in nuclear matter using local high-precision potentials from the Nijmegen group [18] and observed an uncertainty of \pm 0.6 $\mathrm{MeV}$ in the energy per particle at normal density.

In summary, we conclude that the uncertainty arising from the error in the NN data has negligible impact on the many-body system.

Concerning the (long-range) $\pi \mathrm{N}$ LECs, they are likely to impact mostly peripheral partial waves (namely, those high partial waves where no contact terms are present). At NLO, that means $D$-waves and higher, whereas at $\mathrm{N}^{3} \mathrm{LO}$ no contacts are available in $F$-waves and higher. Therefore, one might anticipate that variations of the $\pi \mathrm{N}$ LECs (within the range allowed by $\pi \mathrm{N}$ scattering data) will have only minor impact in nuclear matter, since the sensitivity is limited to peripheral partial waves. Nevertheless, we stress that a systematic investigation with consideration of $\pi \mathrm{N}$ LECs uncertainty consistently in the $2 \mathrm{NF}$ and the $3 \mathrm{NF}$, has not yet been done and is part of our future plans. 


\subsection{Regulator dependence and truncation error in the NN system}

Chiral NN potentials are multiplied by a regulating function, which typically has the form:

$$
f\left(p^{\prime}, p\right)=\exp \left[-\left(p^{\prime} / \Lambda\right)^{2 n}-(p / \Lambda)^{2 n}\right],
$$

where $\Lambda \lesssim 500 \mathrm{MeV}$ has been found to yield favorable perturbative properties. Potentials with different cutoff values constitute a family with similar long-range properties.

Cutoff dependence and truncation error are not to be identified with each other, although, generally, cutoff dependence is expected to decrease with increasing order. Figure 3 shows to which extent this is the case for the two-body system. There, we demonstrate the dependence of NN scattering phase shifts on the chiral order and on the choice of the cutoff scale in the regulating function, Eq. (4.1). The yellow, red and blue bands represent the NLO, $\mathrm{N}^{2} \mathrm{LO}$, and $\mathrm{N}^{3} \mathrm{LO}$ results, respectively, obtained from varying the cutoff between 450 and $600 \mathrm{MeV}$. Although $\mathrm{N}^{2} \mathrm{LO}$ calculations can achieve sufficient accuracy in selected partial waves up to $E_{\text {lab }}=200 \mathrm{MeV}$, only the $\mathrm{N}^{3} \mathrm{LO}$ interactions achieve the level of high-precision potentials, characterized by a $\chi^{2} /$ datum $\sim 1$.

At the two-body level, each time the chiral order is increased, the NN contact terms and/or the two-pion-exchange contributions proportional to the low-energy constants $c_{1,3,4}$ are refitted. We recall that at $\mathrm{N}^{2} \mathrm{LO}$ no new NN contact terms are generated, and therefore improved cutoff independence in the NN phase shifts (compare the yellow and red bands in Fig. 3) is due to changes in the two-pion-exchange contributions. At $\mathrm{N}^{2} \mathrm{LO}$, subleading $\pi \pi N N$ vertices enter into the chiral NN potential. These terms encode the important physics of correlated two-pion-exchange and the excitation of intermediate $\Delta(1232)$ isobar states. Thus, only at this order is it possible to obtain a realistic description of the $\mathrm{NN}$ interaction at intermediate-range, traditionally generated through the exchange of a fictitious $\sigma$ meson of intermediate mass. At $\mathrm{N}^{3} \mathrm{LO}$ in the chiral power counting, 15 additional $\mathrm{NN}$ contact terms (bringing the total to 24 free parameters at $\mathrm{N}^{3} \mathrm{LO}$ ) result in a much improved description of $\mathrm{NN}$ scattering phase shifts.

We observe that, with increasing chiral order, the dependence on the cutoff scale is reduced. In the present work, we employ standard Weinberg power counting in which contributions to the NN potential are computed perturbatively with loop integrals renormalized through counterterms. Weinberg's scheme implicitly assumes that the counterterms introduced to renormalize the perturbative potential are sufficient to also renormalize its nonperturbative resummation, e.g., in the Lippmann-Schwinger equation for computing phase shifts. Kaplan et al. [19], however, pointed out the presence of problems with this assumption, which stimulated intense discussion in the literature [20]. In particular, Nogga et al. [21] performed a systematic investigation of Weinberg's power counting at lowest order and proposed a modified scheme in which contact terms from NLO are promoted to LO to take care of the cutoff dependence in ${ }^{3} P_{0}$ and ${ }^{3} P_{2}$, and from $\mathrm{N}^{3} \mathrm{LO}$ to LO to address the same problem in ${ }^{3} D_{2}$. On the other hand, the consistency problem of Weinberg's power counting appears to be minimal when implemented together with finite-cutoff regularization below the high-energy scale of the EFT. It has been shown [22] that, at a given order, cutoff ranges can be identified where the $\chi^{2}$ of the fit to the $\mathrm{NN}$ data is essentially flat (that is, cutoff independent). In other words, order-by-order renormalization can be accomplished successfully with finite cutoffs in the Weinberg power counting scheme.

In summary, in the NN system we see both excellent order-by-order convergence and reduced cutoff dependence with increasing order. 


\section{Description of the few-nucleon forces}

Before moving to nucleonic matter, in this section we describe the few-nucleon forces as we will apply them in Section 6.

\subsection{The chiral two-body force}

In the present investigation we consider NN potentials at order $\left(q / \Lambda_{\chi}\right)^{2},\left(q / \Lambda_{\chi}\right)^{3}$ and $\left(q / \Lambda_{\chi}\right)^{4}$ in the chiral power counting, where $q$ denotes the small scale set by external nucleon momenta or the pion mass and $\Lambda_{\chi}$ is the chiral symmetry breaking scale. Chiral NN potentials at NLO and $\mathrm{N}^{2} \mathrm{LO}$, corresponding to $\left(q / \Lambda_{\chi}\right)^{2}$ and $\left(q / \Lambda_{\chi}\right)^{3}$, have been constructed previously in Ref. [22] for cutoffs ranging from $\Lambda=450 \mathrm{MeV}$ to about $800 \mathrm{MeV}$. With varying chiral order and cutoff scale, the low-energy constants in the two-nucleon sector are refitted to elastic NN scattering phase shifts and properties of the deuteron. The low-energy constants $c_{1,3,4}$ associated with the $\pi \pi N N$ contact couplings of the $\mathscr{L}_{\pi N}^{(2)}$ chiral Lagrangian are given in Table 1 . We note that the $c_{i}$ can be extracted from $\pi N$ or NN scattering data. The potentials we use here $[23,24]$ follow the second path. At $\mathrm{N}^{2} \mathrm{LO}$, taking the range determined in $\pi N$ analyses as a starting point, values were chosen to best reproduce NN data at that order, see Table 2 of Ref. [24]. At $\mathrm{N}^{3} \mathrm{LO}$, high-precision required a stronger adjustment of $c_{4}$ depending on the regulator function and cutoff. The fitting procedure is discussed in Ref. [24], where it is noted that the larger value for $c_{4}$ has, overall, a very small impact but lowers the ${ }^{3} F_{2}$ phase shift for a better agreement with the phase shift analysis.

In Ref. [22], it was found that the two-body scattering phase shifts can be described well at NLO up to a laboratory energy of about $100 \mathrm{MeV}$, while the $\mathrm{N}^{2} \mathrm{LO}$ potential fits the data up to 200 $\mathrm{MeV}$. Interestingly, in the latter case the $\chi^{2} /$ datum was found to be essentially cutoff independent for variations of $\Lambda$ between 450 and approximately $800 \mathrm{MeV}$. Finally, we also use NN potentials constructed at next-to-next-to-next-to-leading order $\left(\mathrm{N}^{3} \mathrm{LO}\right)$ [24, 23], with low-energy constants $c_{1,3,4}$ as displayed in Table 1 . However, at $\mathrm{N}^{3} \mathrm{LO}$, NN potentials with cutoffs up to $800 \mathrm{MeV}$ are not available. Therefore, in the present study, we limit the cutoff range to 450-600 MeV.

\subsection{The chiral three-nucleon force}

Three-nucleon forces make their appearance at third order in the chiral power counting. They are expressed as the sum of three contributions: the long-range two-pion-exchange part with $\pi \pi N N$ vertex proportional to the low-energy constants $c_{1}, c_{3}, c_{4}$, the medium-range one-pion exchange diagram proportional to the low-energy constant $c_{D}$, and finally the short-range contact term proportional to $c_{E}$. The corresponding diagrams are shown in Fig. 4, labeled as (a), (b), (c), respectively.

Thr natural framework to include 3NFs in the particle-particle ladder approximation to the energy per particle in homogeneous nuclear matter would be the Bethe-Faddeev equation. To facilitate this inclusion, we employ the density-dependent NN interaction derived in Refs. [25, 26] from the $\mathrm{N}^{2} \mathrm{LO}$ chiral three-body force. This effective interaction is obtained by summing one particle line over the occupied states in the Fermi sea. Neglecting small contributions [27] from terms depending on the center-of-mass momentum, the resulting NN interaction can be expressed in analytical form with operator structures identical to those of free-space NN interactions. For symmetric nuclear matter all three-body forces contribute, while for pure neutron matter only terms proportional to the low-energy constants $c_{1}$ and $c_{3}$ are nonvanishing [26, 27]. Previous studies 


\begin{tabular}{|c||c|c|c|c|c|}
\hline NLO & $\Lambda(\mathrm{MeV})$ & $n$ & $c_{1}$ & $c_{3}$ & $c_{4}$ \\
\hline & 450 & 2 & & & \\
& 500 & 2 & & & \\
& 600 & 2 & & & \\
\hline \hline $\mathrm{N}^{2} \mathrm{LO}$ & $\Lambda(\mathrm{MeV})$ & $n$ & $c_{1}$ & $c_{3}$ & $c_{4}$ \\
\hline & 450 & 3 & -0.81 & -3.40 & 3.40 \\
& 500 & 3 & -0.81 & -3.40 & 3.40 \\
& 600 & 3 & -0.81 & -3.40 & 3.40 \\
\hline \hline $\mathrm{N}^{3} \mathrm{LO}$ & $\Lambda(\mathrm{MeV})$ & $n$ & $c_{1}$ & $c_{3}$ & $c_{4}$ \\
\hline & 450 & 3 & -0.81 & -3.40 & 3.40 \\
& 500 & 2 & -0.81 & -3.20 & 5.40 \\
& 600 & 2 & -0.81 & -3.20 & 5.40 \\
\hline \hline
\end{tabular}

Table 1: Values of $n$ and low-energy constants of the dimension-two $\pi N$ Lagrangian, $c_{1,3,4}$, at each order and for each type of cutoff in the regulator function given in Eq. (4.1). None of the $c_{i}$ 's appear at NLO. The low-energy constants are given in units of $\mathrm{GeV}^{-1}$.
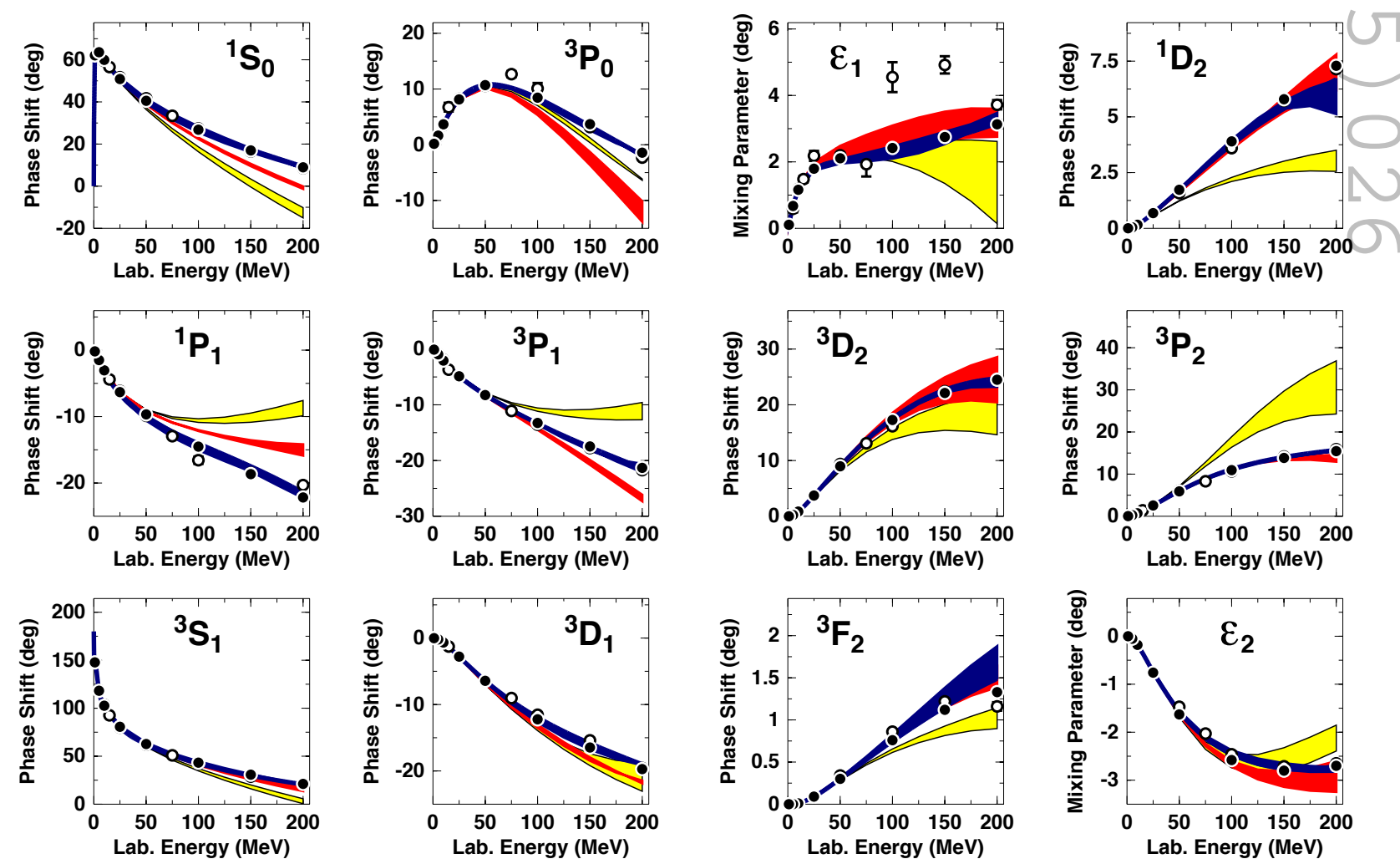

Figure 3: (Color online) Phase shifts for some selected NN partial waves. The yellow, red, and blue bands show the variations of the predictions with changing cutoffs between 450 and 600 at NLO, $\mathrm{N}^{2} \mathrm{LO}$, and $\mathrm{N}^{3} \mathrm{LO}$, respectively. 


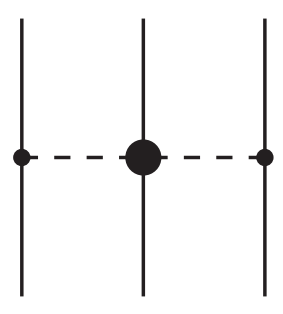

(a)

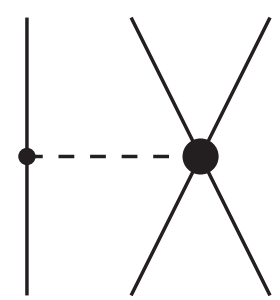

(b)

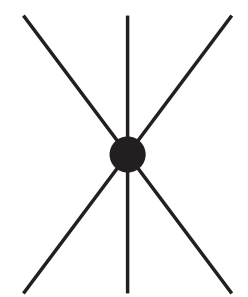

(c)

Figure 4: Diagrams of the $3 \mathrm{NF}$ at $\mathrm{N}^{2} \mathrm{LO}$. See text for more details.

(see e.g., Ref. [5]) have found that 3NF contributions to the energy per particle are dominant at the Hartree-Fock level. When three-body forces are approximated with a density-dependent NN interaction, certain topologies are missing at and beyond second order in perturbation theory. In the case of a pure contact interaction the additional topologies reduce the second-order contribution to the energy per particle by about $50 \%$ at saturation density [28], which corresponds in the present calculation to an uncertainty of $\Delta E / A \lesssim 1 \mathrm{MeV}$ at saturation density.

We fix the low-energy constants $c_{D}$ and $c_{E}$ that appear in the $\mathrm{N}^{2} \mathrm{LO} 3 \mathrm{NF}$ within the threenucleon sector. Specifically, we constrain them to reproduce binding energies of $A=3$ nuclei together with the Gamow-Teller matrix element in tritium $\beta$-decay, following a well established procedure $[29,30,31,32,33,34,35]$. The values of $c_{D}$ and $c_{E}$ are given in Table 2 for the different chiral orders and cutoff scales. We note that the values at $\mathrm{N}^{3} \mathrm{LO}$ in Table 2 are extracted from Refs. $[29,5]$, while those at $\mathrm{N}^{2} \mathrm{LO}$ have been computed in Ref. [13]. Although efforts are in progress to incorporate potentially important $\mathrm{N}^{3} \mathrm{LO} 3 \mathrm{NF}$ contributions [36, 37, 38], both in the fitting procedure and in the neutron and nuclear matter EoS presented in the following section, the current " $\mathrm{N}^{3} \mathrm{LO}$ " study is limited to the inclusion of the $\mathrm{N}^{2} \mathrm{LO}$ three-body force together with the $\mathrm{N}^{3} \mathrm{LO}$ two-body force, an approximation that is commonly used in the literature but whose associated uncertainties have not been carefully analyzed. In Refs. [4, 39], calculations of the neutron matter energy per particle at $\mathrm{N}^{3} \mathrm{LO}$ show a small effect (of about $-0.5 \mathrm{MeV}$ ) at saturation density for the potentials of our purview [24]. The Hartree-Fock contributions to the energy per particle of symmetric nuclear matter from the $\mathrm{N}^{3} \mathrm{LO} 3 \mathrm{NF}$ are attractive and on the order of $7 \mathrm{MeV}$ at saturation density [4]. The inclusion of $3 \mathrm{NFs}$ at $\mathrm{N}^{3} \mathrm{LO}$, however, necessitates a refitting of the $c_{D}$ and $c_{E}$ low-energy constants, which has not yet been performed and would likely result in a smaller change to the total energy per particle at saturation density. Most recently, evidence has been reported [40] that sub-leading terms in the 3NF may provide important contributions to the triton binding energy, as well as indications that similar conclusions apply in symmetric nuclear matter.

In the case of the $\mathrm{N}^{2} \mathrm{LO} N \mathrm{~N}$ interaction with $\Lambda=600 \mathrm{MeV}$, small charge-symmetry-breaking effects have emerged in the fitting procedure. This is visible in Fig. 5, where the $c_{D}-c_{E}$ trajectories which reproduce the experimental ${ }^{3} \mathrm{H}$ and ${ }^{3} \mathrm{He}$ binding energies are displayed. Allowing for chargesymmetry breaking, the values for $c_{E}$ are -0.833 and -0.885 for ${ }^{3} \mathrm{H}$ and ${ }^{3} \mathrm{He}$, respectively. The value shown in Table 2 is the average of these two. The error in the $A=3$ binding energies, when the average value of $c_{E}$ is used, is $\sim 40 \mathrm{keV}$. We do not know at present the origin of this (small) 


\begin{tabular}{|c||c|c|c|}
\hline $\mathrm{N}^{2} \mathrm{LO}$ & $\Lambda(\mathrm{MeV})$ & $c_{D}$ & $c_{E}$ \\
\hline & 450 & -0.326 & -0.149 \\
& 500 & -0.165 & -0.169 \\
& 600 & 0.456 & -0.859 \\
\hline \hline $\mathrm{N}^{3} \mathrm{LO}$ & $\Lambda(\mathrm{MeV})$ & $c_{D}$ & $c_{E}$ \\
\hline & 450 & -0.24 & -0.11 \\
& 500 & 0.0 & -0.18 \\
& 600 & -0.19 & -0.83 \\
\hline
\end{tabular}

Table 2: Values of the $c_{D}$ and $c_{E}$ low-energy constants obtained using the $\mathrm{N}^{2} \mathrm{LO} 3 \mathrm{NF}$ in conjunction with $\mathrm{NN}$ interactions of different orders, for several values of the cutoff $\Lambda$. These constants do not appear at NLO.

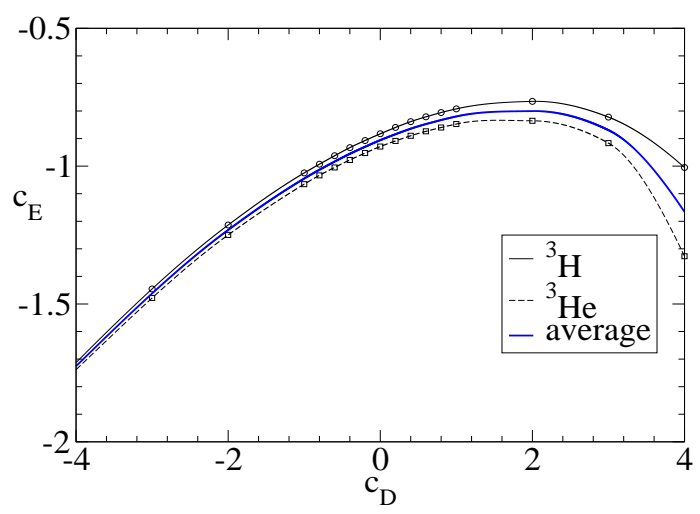

Figure 5: (Color online) $c_{E}-c_{D}$ trajectories fitted to reproduce the experimental ${ }^{3} \mathrm{H}$ and ${ }^{3} \mathrm{He}$ binding energies in the case of the $\mathrm{N}^{2} \mathrm{LO} \mathrm{NN}$ interaction plus $3 \mathrm{NF}$ with $\Lambda=600 \mathrm{MeV}$. The average curve (in blue) is also displayed.

charge-symmetry breaking effect.

\section{Results and discussion}

With the input as described in the previous sections, and in the light of the uncertainty considerations made above, we now move to nuclear and neutron matter.

Our results for the energy per particle as a function of the nuclear density are shown in Fig. 6 for symmetric nuclear matter. We note that the particle-particle ladder approximation employed in the present work is in good agreement with the perturbative results available at $\mathrm{N}^{3} \mathrm{LO}$ from Ref. [5] including up to third-order pp diagrams. In the left panel of Fig. 6, the shaded bands in yellow and red represent the spread of our complete calculations conducted at NLO and $\mathrm{N}^{2} \mathrm{LO}$, respectively. The blue band is the result of a calculation that employs $\mathrm{N}^{3} \mathrm{LO} N \mathrm{~N}$ potentials together with $\mathrm{N}^{2} \mathrm{LO}$ 3NFs. In all cases shown, the cutoff is varied over the range 450-600 MeV. As noted before, the $\mathrm{N}^{3} \mathrm{LO} 3 \mathrm{NFs}$ and $4 \mathrm{NFs}$ are at present omitted, and the resulting convergence pattern gives an estimate on the theoretical uncertainty of the calculation (and not necessarily of the chiral EFT expansion per se). On the right-hand side of the figure, the individual curves corresponding to each 
order and cutoff are displayed. We observe that at NLO the potentials constructed at lower cutoff scales do not exhibit saturation until very high densities. On the other hand, for the $600 \mathrm{MeV}$ cutoff potential the ${ }^{1} S_{0}$ partial wave (together with the ${ }^{3} S_{1}$ partial wave) is sufficiently repulsive to enable saturation at a relatively smaller density. We observe that the convergence pattern for the low-cutoff $(\Lambda=450-500 \mathrm{MeV})$ potentials is significantly better than for the $600 \mathrm{MeV}$ potential. Overall there is a large spread from cutoff variations both at NLO and $\mathrm{N}^{2} \mathrm{LO}$ beyond nuclear matter saturation density. Moreover, the bands at these two orders do not overlap, suggesting that their width is not a suitable representation of the uncertainty. Although the (incomplete) $\mathrm{N}^{3} \mathrm{LO}$ calculation reveals a strong reduction of the cutoff dependence, it is important to notice that an uncertainty of about $8 \mathrm{MeV}$ remains at saturation density. While we do not expect much of a change in nuclear matter predictions from $4 \mathrm{NFs}[41,42,4]$, it is quite possible that the inclusion of $\mathrm{N}^{3} \mathrm{LO} 3 \mathrm{NFs}$ might reduce either the cutoff dependence or improve the convergence pattern. This will be an interesting subject for future investigations.

The results for neutron matter are presented in Fig. 7, where the left and right panels have the same meaning as in Fig. 6. Note that the range of densities under consideration is smaller for neutron matter in order to keep the Fermi momentum below the cutoff in all cases. We see a large spread at NLO for the largest densities considered, whereas the band has only moderate size at the next order and remains small for our $\mathrm{N}^{3} \mathrm{LO}$ calculation. Similar to what was observed in symmetric nuclear matter, the bands at $\mathrm{NLO}$ and $\mathrm{N}^{2} \mathrm{LO}$ do not overlap in neutron matter. In additional the $\mathrm{N}^{3} \mathrm{LO}$ band does not generally overlap with the $\mathrm{N}^{2} \mathrm{LO}$ band. Therefore, the variation obtained by changing the cutoff does not seem to provide a reliable representation of the uncertainty at the given order. A better way to estimate such uncertainty is to consider the difference between the predictions at two consecutive orders.

In Fig. 8 we present the results for the symmetry energy $E_{\text {sym }}$, which is defined as the strength of the quadratic term in an expansion of the energy per particle in asymmetric matter with respect to the asymmetry parameter $\alpha$ :

$$
\bar{E}(\rho, \alpha) \approx \bar{E}(\rho, \alpha=0)+E_{\mathrm{sym}} \alpha^{2}+\mathscr{O}\left(\alpha^{4}\right)
$$

where $\bar{E}=E / A$ is the energy per particle and $\alpha=\left(\rho_{n}-\rho_{p}\right) /\left(\rho_{n}+\rho_{p}\right)$. The nearly linear behavior of $\bar{E}(\rho, \alpha)$ with $\alpha^{2}$ has been confirmed by many microscopic calculations (see for instance Ref. [43] and more recently Refs. [44, 45]). It justifies the common approximation of neglecting powers beyond $\alpha^{2}$ in the expansion above and thus defining the symmetry energy as the difference between the energy per particle in neutron matter and symmetric nuclear matter.

As mentioned in Sec. 2, systematic efforts are ongoing to set better empirical constraints on the symmetry energy, through both laboratory and astrophysical measurements. It is therefore important to have an understanding of the theoretical uncertainty affecting calculations of this quantity. The spread due to the change of the cutoff values in our NLO, $\mathrm{N}^{2} \mathrm{LO}$, and $\mathrm{N}^{3} \mathrm{LO}$ calculations is represented by the three bands as before. As observed previously for symmetric matter, the spread due to cutoff variations remains large at $\mathrm{N}^{2} \mathrm{LO}$, with some minimal overlap with the NLO band. The $\mathrm{N}^{3} \mathrm{LO}$ band reflects the large cutoff sensitivity previously observed in symmetric matter. Again, we conclude that the spread generated by changing the cutoff does not in general provide a reliable estimate of the theoretical uncertainty. 

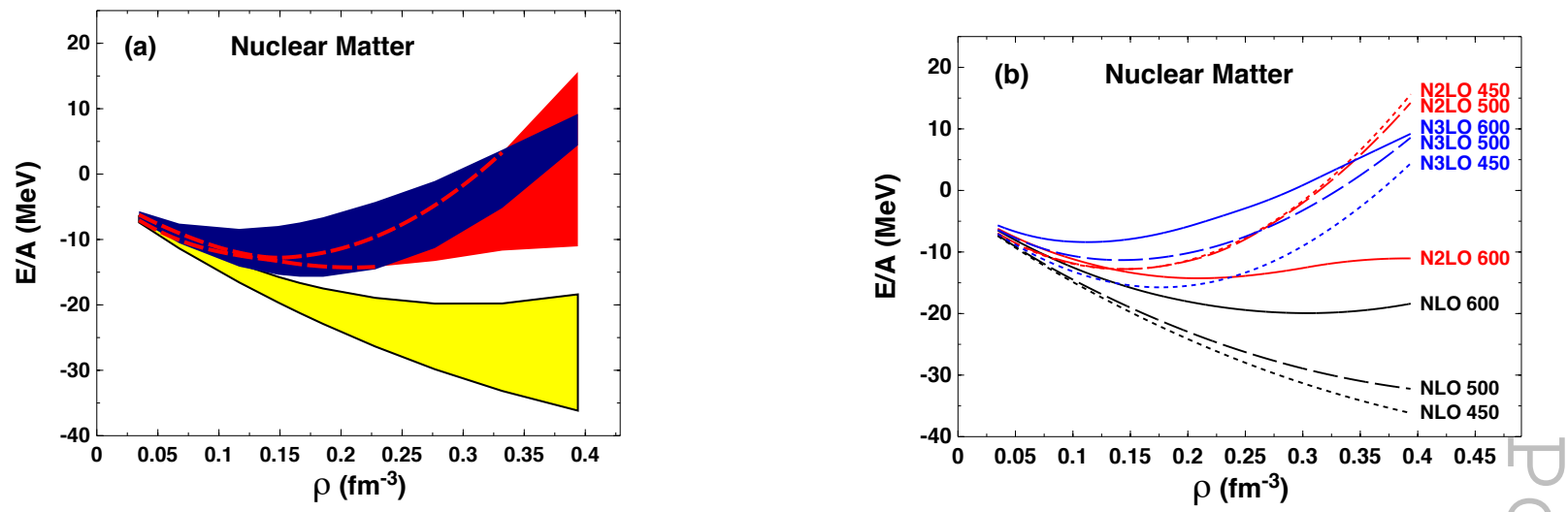

Figure 6: (Color online) Energy/nucleon (E/A) in symmetric nuclear matter as a function of density, $\rho$. Left frame: The yellow and red bands represent the uncertainties in the predictions due to cutoff variations as obtained in complete calculations at $\mathrm{NLO}$ and $\mathrm{N}^{2} \mathrm{LO}$, respectively. The blue band is the result of a calculation employing $\mathrm{N}^{3} \mathrm{LO}$ NN potentials together with $\mathrm{N}^{2} \mathrm{LO} 3 \mathrm{NFs}$. The dashed lines show the upper or lower limits of hidden bands. Right frame: predictions at the specified order and cutoff value.
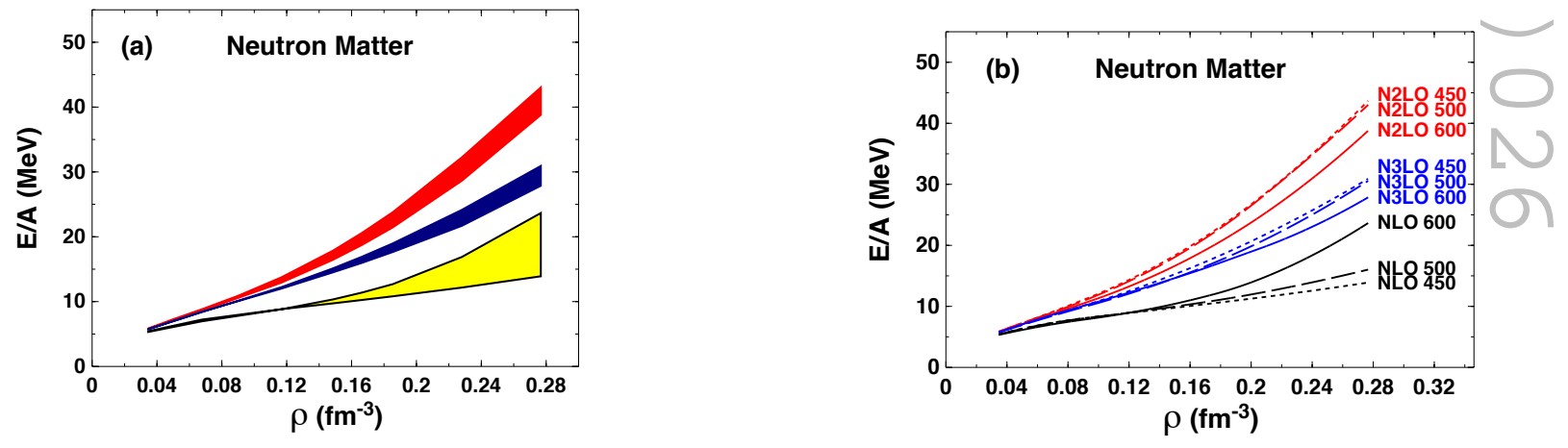

Figure 7: (Color online) As in Fig. 6 for pure neutron matter.

\section{Conclusions and future plans}

We have reported predictions for the energy per particle in symmetric nuclear matter and pure neutron matter, focusing on uncertainties related to order-by-order convergence. Compared to the consistent NLO and $\mathrm{N}^{2} \mathrm{LO}$ results for the EoS, which themselves exhibit relatively little overlap even for a large spread of momentum-space cutoffs $\Lambda=450-600 \mathrm{MeV}$, the results from employing $\mathrm{N}^{3} \mathrm{LO}$ two-nucleon and $\mathrm{N}^{2} \mathrm{LO}$ three-nucleon forces imply non-negligible uncertainties associated with missing higher-order terms in the chiral expansion. We find that the uncertainty associated with the cutoff variation is generally larger in symmetric nuclear matter than in pure neutron matter, but in the latter case we find that the results from one chiral order to the next have little overlap. This suggests that further systematic studies of the order-by-order convergence should be performed, together with variations in the resolution scale and low-energy constants, 


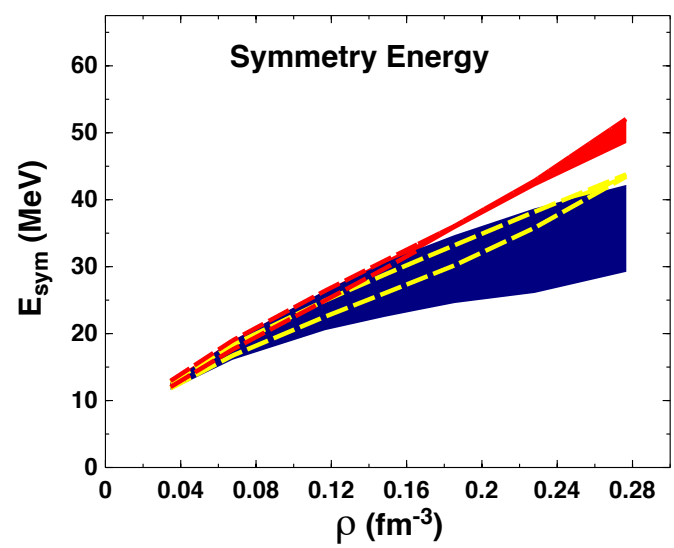

Figure 8: (Color online) The symmetry energy, $E_{\mathrm{sym}}$, as a function of density, $\rho$. Meaning of bands and dashed lines as in the left panel of Fig. 6.

to accurately estimate the complete theoretical uncertainties in chiral EFT predictions of nuclear many-body systems. The two- and three-body potentials considered in the present work can serve as a basis for such future uncertainty estimates.

In the near future, we wish to make the analysis broader and more systematic. In particular, we plan to extend the present study by considering variations of the $\pi \mathrm{N}$ LECs within the uncertainty allowed by the $\pi \mathrm{N}$ analyses. For each set of LECs, we will construct a corresponding NN potential and consistently adjust the $3 \mathrm{NF}$ by refitting its parameters to the three-nucleon system.

Furthermore, it may be of interest to look at the 5th order $\left(\mathrm{N}^{4} \mathrm{LO}\right)$ of chiral EFT. Based upon Ref. [46], a high-precision NN potential at $\mathrm{N}^{4} \mathrm{LO}$ will soon become available. Concerning threenucleon forces at $\mathrm{N}^{4} \mathrm{LO}$, there are sizeable pion-exchange 3NFs [47] and, furthermore, a new set of ten $3 \mathrm{NF}$ contact interactions appear [48]. Thus, the fifth order may still play an important role in the chiral expansion.

\section{Acknowledgments}

Support from the U.S. Department of Energy Office of Science, Office of Basic Energy Science, under Award Nos. DE-FG02-03ER41270 and DE-FG02-97ER41014 is acknowledged. Part of the results presented here have been obtained at the INFN-Pisa computer center.

\section{References}

[1] S. Weinberg, Nonlinear realization of chiral symmetry, Phys. Rev. 166 (1968) 1568.

[2] S. Weinberg, Phenomenological Lagrangians, Physica 96A (1979) 327.

[3] L. Coraggio, J. W. Holt, N. Itaco, R. Machleidt and F. Sammarruca, Reduced regulator dependence of nuclear-matter predictions with perturbative chiral interactions, Phys. Rev. C 87 (2013) 014322.

[4] T. Krüger, I. Tews, K. Hebeler, and A. Schwenk, Neutron matter from chiral effective field theory interactions, Phys. Rev. C 88 (2013) 025802. 
[5] L. Coraggio, J. W. Holt, N. Itaco, R. Machleidt, L. E. Marcucci, and F. Sammarruca, Nuclear-matter equation of state with consistent two- and three-body perturbative chiral interactions, Phys. Rev. C 89 (2014) 044321.

[6] C. Wellenhofer, J. W. Holt, N. Kaiser and W. Weise, Nuclear Thermodynamics from chiral low-momentum interactions, Phys. Rev. C 89 (2014) 064009.

[7] Z.H. Li, U. Lombardo, H.-J. Schulze, and W. Zuo, Consistent nucleon-nucleon potentials and thee-body forces, Phys. Rev. C 77 (2008) 034316.

[8] F. Sammarruca, Analysis of the symmetry energy in a microscopic approach, Int. J. Mod. Phys. E 22 (2014) 1330031, and references therein.

[9] R. J. Furnstahl, D. R. Phillips, and S. Wesolowski, A recepy for EFT uncertainty quantification in nuclear physics, J. Phys. G 42 (2015) 034028.

[10] S. K. Bogner, A. Schwenk, R. J. Furnstahl, and A. Nogga, Is nuclear matter perturbative with low-momentum interactions?, Nucl. Phys. A 763 (2005) 59.

[11] K. Hebeler, S. K. Bogner, R. J. Furnstahl, A. Nogga, and A. Schwenk, Improved nuclear matter calculations from chiral low-momentum interactions, Phys. Rev. C 83 (2011) 031301.

[12] A. Gezerlis, I. Tews, E. Epelbaum, S. Gandolfi, K. Hebeler, A. Nogga, and A. Schwenk, Quantum Monte Carlo calculations with chiral effective field theory interactions, Phys. Rev. Lett. 111 (2013) 032501.

[13] F. Sammarruca, L. Coraggio, J. W. Holt, N. Itaco, R. Machleidt, L. E. Marcucci, Towards order-by-order calculations of the nuclear and neutron matter equations of state in chiral effective field theory, Phys. Rev. C 91 (2015) 054311.

[14] M.B. Tsang et al., Constraints on the symmetry energy and neutron skins from experiments and theory, Phys. Rev. C 86 (2012) 015803.

[15] G. Baardsen et al., Coupled-cluster studies of infinite nuclear matter, Phys. Rev. C 88 (2013) 054312.

[16] G. Hagen et al., Coupled-cluster calculations of infinite nucleonic matter, Phys. Rev. C 89 (2014) 014319.

[17] R. Navarro Perez, E. Garrido, J.E. Amaro, and E. Ruiz Arriola Triton binding energy with realistic precision, Phys. Rev. C 90 (2014) 047001.

[18] V.G.J. Stoks, R.A.M. Klomp, C.P.F. Terheggen, and J.J. de Swart Construction of high-quality NN potential models, Phys. Rev. C 49 (1994) 2950.

[19] D.B. Kaplan, M.J. Savage, and M.B. Wise, Nucleon-nucleon scattering from effective field theory, Nucl. Phys. B478 (1996) 629; A new expansion for nucleon-nucleon interactions, Phys. Lett. B 424 (1998) 390; Two-nucleon systems from chiral effective theory, Nucl. Phys. B534 (1998) 329.

[20] See Refs. [146-164] in Ref. [24].

[21] A. Nogga, R.G.E. Timmermans, and U. van Kolck, Renormalization of one-pion exchange and power counting, Phys. Rev. C 72 (2005) 054006.

[22] E. Marji, A. Canul, Q. MacPherson, R. Winzer, Ch. Zeoli, D.R. Entem, and R. Machleidt, Nonperturbative renormalization of the chiral nucleon-nucleon interaction up to next-to-next-to-leading order, Phys. Rev. C 88 (2013) 054002.

[23] D.R. Entem and R. Machleidt, Accurate charge-dependent nucleon-nucleon potentials at fourth order of chiral perturbation theory, Phys. Rev. C 68 (2003) 041001. 
[24] R. Machleidt and D.R. Entem, Chiral effective field theory and nuclear forces, Phys. Rep. 503 (2011) 1 .

[25] J. W. Holt, N. Kaiser, and W. Weise, Chiral three-nucleon interaction and the ${ }^{14} C$-dating $\beta$ decay, Phys. Rev. C 79 (2009) 054331.

[26] J. W. Holt, N. Kaiser, and W. Weise, Density-dependent effective nucleon-nucleon interaction from chiral three-nucleon forces, Phys. Rev. C 81 (2010) 024002.

[27] K. Hebeler and A. Schwenk, Chiral three-nucleon forces and neutron matter, Phys. Rev. C 82 (2010) 014314.

[28] N. Kaiser, Exact calculation of three-body contact interaction to second order, Eur. Phys. J. A 48 (2012) 58 .

[29] L.E. Marcucci, A. Kievsky, S. Rosati, R. Schiavilla, and M. Viviani, Chiral effective field theory predictions for muon capture on deuteron and ${ }^{3}$ He, Phys. Rev. Lett. 108 (2012) 052502.

[30] A. Gardestig and D.R. Phillips, How low-energy weak reactions can constrain three-nucleon forces and the neutron-neutron scattering length, Phys. Rev. Lett. 96 (2006) 232301.

[31] D. Gazit, S. Quaglioni, and P. Navratil, Three-nucleon low-energy constants from the consistency of interactions and currents in chiral effective field theory, Phys. Rev. Lett. 103 (2009) 102502.

[32] M. Viviani, L. Girlanda, A. Kievsky, and L.E. Marcucci, Effect of three-nucleon interactions in $p-{ }^{3}$ He elastic scattering, Phys. Rev. Lett. 111 (2013) 172302.

[33] M. Piarulli, L. Girlanda, L.E. Marcucci, S. Pastore, R. Schiavilla, and M. Viviani, Electromagnetic structure of $A=2$ and 3 nuclei in chiral effective field theory, Phys. Rev. C 87 (2013) 014006.

[34] L.E. Marcucci, R. Schiavilla, and M. Viviani, Proton-proton weak capture in chiral effective field theory, Phys. Rev. Lett. 110 (2013) 192503.

[35] M. Viviani, A. Baroni, L. Girlanda, A. Kievsky, L.E. Marcucci, and R. Schiavilla, Chiral effective field theory analysis of hadronic parity violation in few-nucleon systems, Phys. Rev. C 89 (2014) 064004.

[36] S. Ishikawa and M. R. Robilotta, Two-pion exchange three-nucleon potential: $O\left(q^{4}\right)$ chiral expansion, Phys. Rev. C 76 (2007) 014006.

[37] V. Bernard, E. Epelbaum, H. Krebs, and U.-G. Meissner, Subleading contributions to the chiral three-nucleon force: Long-range terms, Phys. Rev. C 77 (2008) 064004.

[38] V. Bernard, E. Epelbaum, H. Krebs, and U.-G. Meissner, Subleading contributions to the chiral three-nucleon force. II. Short-range terms and relativistic corrections, Phys. Rev. C 84 (2011) 054001.

[39] I. Tews, T. Krüger, K. Hebeler, and A. Schwenk, Neutron matter at next-to-next-to-next-to-leading order in chiral effective theory, Phys. Rev. Lett. 110 (2013) 032504.

[40] K. Hebeler et al., Efficient calculation of chiral three-nucleon forces up to N3LO for ab initio studies, Phys. Rev. C 91 (2015) 044001.

[41] E. Epelbaum, Four-nucleon forces using the method of unitary transformation, Eur. Phys. J. A 34 (2007) 197.

[42] D. Rozpedzik et al., A first estimation of chiral four-nucleon force effects in 4 He, Acta Phys. Polon. B 37 (2006) 2889.

[43] I. Bombaci and U. Lombardo, Asymmetric nuclear matter equation of state, Phys. Rev. C 44 (1991) 1892. 
[44] D. Alonso and F. Sammarruca, Microscopic calculations in asymmetric nuclear matter, Phys. Rev. C 67054301 (2003) 054301.

[45] C. Drischler, V. Soma, and A. Schwenk, Microscopic calculations and energy expansions for neutron-rich matter, Phys. Rev. C 89 (2014) 025806.

[46] D.R. Entem, N. Kaiser, R. Machleidt, and Y. Nosyk, Peripheral nucleon-nucleon scattering at fifth order of chiral perturbation theory, Phys. Rev. C 91 (2015) 014002.

[47] H. Krebs, A. Gasparian, and E. Epelbaum, Chiral three-nucleon forces at $N^{4} L O$ : Longest range contributions, Phys. Rev. C 85 (2012) 054007.

[48] L. Girlanda, A. Kievsky, and M. Viviani, Subleading contributions to the three-nucleon contact interaction, Phys. Rev. C 84 (2011) 014001. 